miệng vai trò do hệ thống đệm của nước bọt. Xylitol bản thân nó không cung cấp thêm các thành phần của hệ đệm (bicarbonate, phosphate...) mà nó kích thích các tuyến nước bọt tiết ra nhiều hơn để trung hòa axid trong miệng, tạo ra sản phẩm là CO2 thoát ra khỏi khoang miệng và kết quả là loại bỏ axid, chống lại sâu răng (Ericsson, 2007). Vai trò của pH liên quan đến chỉ số sâu mất trám thể hiện rõ rệt qua nghiên cứu của Ahlam T. Al-Mashhadani và cộng sự (2005) với kết quả pH từ 7,7-8,1 có chỉ số DMFS thấp hơn đáng kể so với nhóm có pH từ 6,9-7,3. Nghiên cứu của $M$ Ribelles Llop và cộng sự (2010) khẳng định thêm tác dụng nhai kẹo xylitol giúp kích thích dòng chảy, phục hồi $\mathrm{pH}$ và giảm mức S.mutan trong nước bọt. Vì vậy, ảnh hưởng của xylitol lên pH nước bọt với tác động tăng $\mathrm{pH}$ đáng kể có vai trò quan trọng trong phòng ngừa sâu răng.

\section{KẾT LUÂ̂N}

Sử dụng kẹo xylitol có hiệu quả tăng lưu lượng nước bọt và tăng pH nước bott, tuy nhiên, cũng cần đánh giá tại nhiều thời điểm hơn để xem khả năng duy trì hiệu quả của phương pháp này.
TÀI LIỆU THAM KHẢO

1. Leone CW, Oppenheim FG. Physical and chemical aspects of saliva as indicators of risk for dental caries in humans. J Dent Educ. 2001; 65: 1054-1062.

2. Hegde $\mathbf{M N}$, Attavar SH, Shetty $\mathbf{N}$ et al. Saliva as a biomarker for dental caries: A systematic review. J Conserv Dent. 2019; 22(1): 2-6.

3. Sok JJ, Jaspreet B,Paul I et al. Oral and Systemic Effects of Xylitol Consumption. Caries Res. 2019; 9(53): 491-501.

4. Ralph M. Duckworth, S. Jones. On the relationship between the rate of salivary flow and salivary fluoride clearance. Caries Res. 2015; 49: 141-146.

5. Israa Mohammed Dawood, Sulafa K. ElSamarra. Saliva and Oral Health. International Journal of Advanced Research in Biological Science. 2018; 5(7): 1-45.

6. Sawair F., Kosti O., Getting S., and Renshaw D. Saliva: Physiology and diagnostic potential in health and disease. Scientific World Jornal, 2010; 10: 11-12

7. Autio JT, Courts FJ. Acceptance of the xylitol chewing gum regimen by preschool children and teachers in a Head Start program: a pilot study. Pediatric Dentistry. 2001; 23(1): 71-74.

8. C Fenoll - Palomares at al. Unstimulated salivary flow rate, $\mathrm{pH}$ and buffer capacity of saliva in healthy volunteers. Revista espanola de enfermedades digestivas: organo oficial de la Sociedad Espanola de Patologia Digestiva. 2004; 96(11):773-83

\title{
KHẢO SÁT TÌNH HÌNH SỬ DUNG ĐIÊN THOAI THÔNG MINH Ở SINH VIÊN ĐAI HỌC TRÊN ĐİA BÀN HÀ NộI BẰNG THANG ĐIỂM ĐÁNH GIÁ NGHIỆN ĐIỆN THOẠI THÔNG MINH PHIÊN BẢN RÚT GỌN
}

\author{
Đinh Trọng Hà*, Quản Minh Anh*, \\ Nguyễn Thị Hoa*, Nguyễn Lê Chiến* Trần Hải Anh*
}

\section{TÓM TẮT}

Mục tiêu: Đánh giá một số đặc điểm xã hội hoc về sử dụng điện thoại thông minh ở những sinh viển đại học trên địa bàn Hà Nội. Đối tượng và phương phã́p: Nghiên cứu mô tả cắt ngang được tiến hành ngấu nhiên trên 1314 sinh viên năm 2-4 tại 36 trường đại học trên đia bàn thành phố Hà Nội. Các đối tượng được hướng dẫn trả lời trực tuyến bộ câu hỏi sử dụng thang đo nghiện điện thoai thông minh phiên bản rút gọn trên Google Form. Kểt quả: Trong các đối tượng tham gia, nữ giới chiếm ưu thế $(71,61 \%)$ và sinh viên tuổi từ 18-21 chiếm phân lớn. Tỳ lệ sinh viên nghiện sử dung điên thoai thông minh là $55,56 \%$, trong đó tuổi 18-20 chiếm tỷ lệ cao hơn các lứa tuổi khác. Bắt

*Hoc viện Quân y

Chịu trách nhiệm chính: Trân Hải Anh

Email: anhhtr@yahoo.com

Ngày nhận bài: $15 / 3 / 202$

Ngày phản biên khoa hoc: 12/4/2021

Ngày duyệt bài: 7/5/2021 đâu sử dụng điện thoại thông minh của sinh viên tập trung chủ yếu ở tuổi 12-18. Phần lớn sinh viên trong nghiên cứu này sử dụng ứng dụng Facebook và Facebook Messenger trên điện thoại thông minh. Sinh viên nghiện điện thoại thông minh có tần suất tập thể dục và tham gia làm thêm thấp hơn so với nhóm không nghiện. Kết luận: Tỷ lệ sinh viên trên địa bàn Hà Nô̂i nghiện sử dung điên thoai thông minh cao và những khác biệt giữa sinh viển nghiện và không nghiên điên thoại thông minh về các yếu tố xã hội hoc là một thực trạng đáng lo ngại, đó cũng là dấu hiệu cảnh báo cần thiết để tìm kiếm các biện pháp nghiển cứu và can thiệp ở cấp độ rộng hơn cho hiện trạng nghiên này.

Từ khóa: Điện thoại thông minh, nghiện điện thoại thông minh, sinh viển.

\section{SUMMARY \\ EVALUATION OF SMARTPHONE USAGE AMONG STUDENTS IN HANOI USING SMARTPHONE ADDICTION SCALE-SHORT VERSION (SAS-SV)}


Objectives: Evaluate socio-demographic characteristics on smartphone usage among students in Hanoi. Subjects and methods: A descriptive cross-sectional study conducted randomly on 1314 students of the second to fourth year registered from 36 universities in Hanoi. Subjects were instructed to answer a set of Smartphone Addiction Scale - Short Version (SAS-SV) questionaries online using Google Forms. Results: Among subjects, females dominated $(71.61 \%)$, and the majority of the participated students aged 18-21 years old. The percentage of students with smartphone addiction was $55.56 \%$, in which students of 18-20 years old was higher than other ages. For most students, smartphone usage began when they were around 12-14 years old. Most participated students had been using Facebook and Facebook Messenger applications on their smartphone. Smartphone addicted students did less physical exercises and part-time works compared to their non-addicted counterparts. Conclusion: Smartphone addiction is prevalent in university students in Hanoi, and differences in sociodemographic features between smartphone addicted and non-addicted students are considered as worrying, which are also alarming signs for searching solutions for researching and intervening at a wider scope in this addition state.

Key words: Smartphones, smartphone addiction, students

\section{I. ĐẶT VẤN ĐỀ}

Điện thoại thông minh là một thiết bị di động kết hợp các chức năng của máy tính và điện thoại cầm tay, có thể được sử dụng như một máy tính nhỏ và kết nối với internet cùng các hoạt động ứng dụng đa phương tiện [1]. Bởi sự tiện lợi từ tích hợp chức năng, việc sử dụng điện thoại thông minh ngày càng tăng và trở thành một phần không thể thiếu trong cuộc sống của khá nhiều người ở các châu lục [1], [4], [5]. Tuy nhiên việc tập trung quá nhiều vào điện thoại thông minh có thể dẫn đến chứng nghiền hành vi, có liên quan đến một số nguy cơ sức khỏe, từ rối loạn tâm lý xã hội như lo lắng, trầm cảm [1] và các vấn đề xã hội [1], [2]. Đánh giá về nghiện điện thoại thông minh sử dụng "Thang điểm nghiện điện thoại thông minh" (Smartphone Addiction Scale, SAS) được phát triển bằng tiếng Hàn Quốc và tiếng Anh bao gồm 33 mục, sau đó có phiên bản rút gọn (Short Version, SV) gồm 10 mục [2]. Phiên bản SAS-SV được dùng rộng rãi nhất với các bản dịch sang nhiều thứ tiểng [4], [5], làm nó trở thành một công cụ hữu ích trong việc đánh giá nghiện điện thoại thông minh theo các khía cạnh xã hội học. Tỷ lệ người sở hữu điện thoại thông minh ở Việt Nam cao, lên tới $72 \%$ và tỷ lệ sở hữu điện thoại và tìm kiếm thông tin trên mạng bằng loại điện thoại này lên đến $68 \%$ [3], tuy nhiên tại Việt nam việc áp dụng phiên bản SAS-SV bằng tiếng Việt vẫn chưa được phổ biến. Việc sử dụng thang điểm SAS-SV để khảo sát tình trạng sư dung điện thoai thông minh trong đối tương sinh viên đại học là những nghiên cứu cần thiết để có biện pháp can thiệp xã hội sớm. Do đó, chúng tôi tiến hành nghiên cứu này nhằm đánh giá một số đặc điểm xã hội học về sử dụng điện thoại thông minh ở những sinh viên đại học trên địa bàn Hà Nội.

\section{II. ĐỐI TƯợNG VÀ PHƯƠNG PHÁP NGHIÊN CỨU \\ 2.1. Đối tượng và thời gian nghiên cứu:} 1314 sinh viên các năm 2-4, tuổi từ 18-25, ở 36 trường đại học trên địa bàn thành phố Hà Nội. Nghiên cứu được tiến hành từ tháng 6 đến tháng 10/2020.

2.2. Phương pháp nghiên cứu: Nghiên cứu mô tả cắt ngang được tiến hành ngẫu nhiên trên các sinh viên, sử dụng phiên bản rút gọn SAS-SV được dịch sang tiếng Viêtt với 10 muc. Sử dụng thang đo Likert để cho điểm ở mỗi cẩu hỏi về mức độ tán thành từ 1-6 điểm tương ứng với các mức độ từ "Rất không đồng ý" đến "Hoàn toàn đồng 'y". Đánh giá nghiện sử dụng điện thoại khi điểm từ 31 trở lên ở nam và từ 33 điểm trở îen ở nữ [2]. Tất cả các yếu tố quan tâm trên được tao ở dang câu hỏi trên Google Form và đưa lên các diễn đàn sinh viên của các trường đại học, các đối tượng nghiên cứu sẽ trực tiếp trả lời duy nhất một lần các câu hỏi thông qua việc đăng nhập theo link:

https://docs.google.com/forms/d/16DoU8B2 WK9eMZpBVc7tjo76RihevLkU4-

3fIV8rsG3c/closedform

2.3. Xử lý số liệu: So sánh giữa các chỉ số thu được ở hai nhóm đối tượng nghiện và không nghiện điện thoại thông minh trong nghiên cứu này sử dụng phần mềm SPSS 20 với các kiểm định phù hợp. Mức xác định sự khác biệt có ý nghĩa thống kê trong các kiểm định là 0,05.

\section{KẾT QUẢ NGHIÊN CỨU VÀ BÀN LUẬN}

Các đặc điểm về phân bố giới và tuổi của đối tượng nghiên cứu được trình bày trên các bảng 1 và 2 .

Bảng 1. Phân bố về giới của đôii tượng nghiên cứu.

\begin{tabular}{|c|c|c|}
\hline Đối tượng & Số lượng (n) & Tỷ lệ (\%) \\
\hline Nam & 373 & 28,39 \\
\hline Nữ & 941 & 71,61 \\
\hline Tống & $\mathbf{1 3 1 4}$ & $\mathbf{1 0 0}$ \\
\hline
\end{tabular}

Qua bảng 1 cho thây trong số các sinh viên tham gia vào nghiên cứu nữ giới chiếm ưu thế $(71,61 \%)$. 
Bảng 2. Phân bố về tuổi của đối tượng nghiên cứu.

\begin{tabular}{|c|c|c|}
\hline Tuối & Số lượng (n) & Tỷ lệ (\%) \\
\hline $\mathbf{1 8 - 1 9}$ & 527 & 40,10 \\
\hline $\mathbf{2 0 - 2 1}$ & 581 & 44,22 \\
\hline $\mathbf{2 2 - 2 3}$ & 155 & 11,80 \\
\hline $\mathbf{2 4 - 2 5}$ & 51 & 3,88 \\
\hline Tống & $\mathbf{1 3 1 4}$ & $\mathbf{1 0 0}$ \\
\hline
\end{tabular}

Kết quả trên bảng 2 cho thấy sinh viên tham gia nghiên cứu chiếm số lượng lớn ở độ 18-21 tuổi $(84,22 \%)$. Đây là lứa tuổi của những năm đâu tới năm ba đai học.

Tỷ lệ nghiện-không nghiện điện thoại thông minh và các tỷ lệ đó phân bố theo tuổi được trình bày trên hình 1.

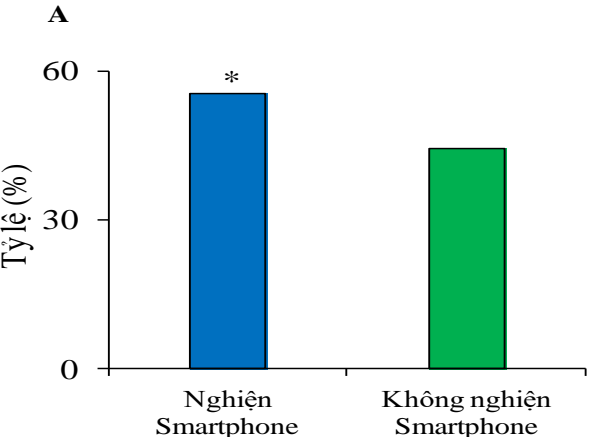

Hình 1. Tỷ lệ sinh viên nghiện điện thoại thông minh (A) và tỷ lệ sinh viên nghiện điện thoại thông minh theo tuôi (B). (*: $p<0,05$, kiêm định $t$ ).

Trên hình $1 \mathrm{~A}$ cho thấy tỷ lê sinh viên nghiên sử dụng điện thoại thông minh $(55,56 \%)$ lớn hơn so với tỷ lệ không nghiện $(44,44 \%)$ trên tổng số 1314 sinh viên $(p<0,05)$. Sinh viên nghiện điện thoại thông minh chiếm tỷ lệ cao ở tuổi 18-20 $(p<0,05)$, không có sư khác biêt về tỷ lệ nghiện và không nghiện ở tuổi 21-24, trong

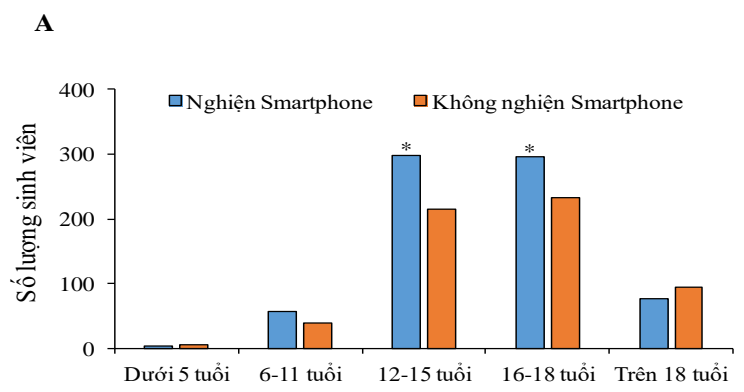

khi ở tuổi 25 sinh viên không nghiên điên thoai thông minh lại có tỷ lệ cao $(p<0,05)$ (Hình $1 B)$.

Các đặc điểm liên quan tới thời điểm bắt đâu sử dụng và các ứng dụng cài đặt trên điện thoại thông minh sinh viên hay dùng được trình bày trên Hình 2.

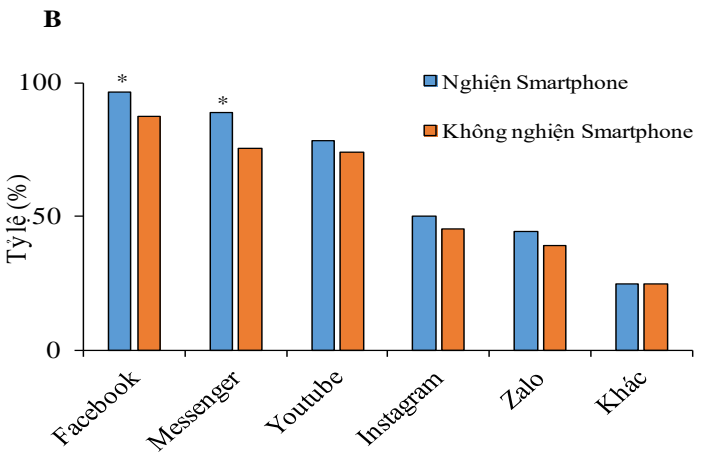

Hình 2. Thời điểm băt đầu sử dụng điện thoại thông minh (A) và các ứng dụng trên Smartphone sinh viên hay dùng (B). (*: $p<0,05$, kiểm định $t)$.

Hình 2A cho thấy tuổi bắt đâu sử dụng điện thoai thông minh của sinh viên tâp trung ở độ 12-18 tuổi. Số sinh viên nghiện điện thoại thông minh bắt đầu sử dụng chúng ở lứa tuổi 12-18 lớn hơn so với ở sinh viên không nghiện $(\mathrm{p}<$ 0,05 ). Phần lớn sinh viên trong nghiên cứu này sử dụng ứng dụng Facebook và Facebook Messenger (Hình 2B), trong đó sinh viên nghiện điện thoại thông minh sử dụng mạng xã hội Facebook và ứng dụng Messenger nhiều hơn so

Kết quả về liên quan sử dụng điện thoại thông minh và tham gia họat động thể dục thể thao/làm thêm được trình bày trền hình 3.

Hình $3 \mathrm{~A}$ cho thấy sinh viên nghiện điện thoại thông minh có tần suất tập thể dục thấp hớn so với sinh viên không nghiện chúng $(p<0,05-<0,01)$. Sinh viên nghiện điện thoại thông minh cũng đi làm thêm ít hơn so với sinh viên không nghiện điện thoại thông minh ( $p<0,05-<0,01)$ (Hình 3B). 
$\mathbf{A}$

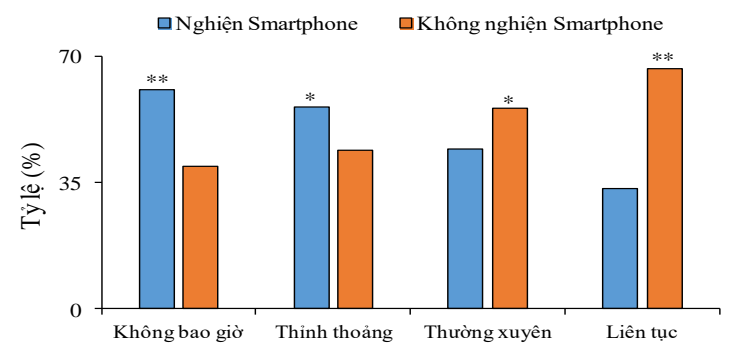

B

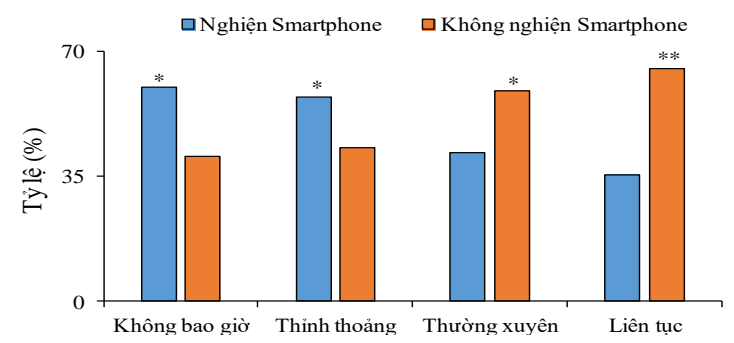

Hình 3. Tỷ lệ sinh viên sử dụng điện thoại thông minh tham gia hoạt động thể dục thể thao (A) và làm thêm (B). (*: $p<0,05 ;$ **: $p<0,01$; kiểm định t).

\section{BÀN LUẬN}

4.1. Tỷ iệ sinh viên nghiện điện thoại thông minh. Sử dụng bộ câu hỏi SAS-SV phiển bản tiếng Việt đánh giá nghiện sử dụng điên thoại thông minh ở 1314 sinh viên trên địa bàn Hà Nội, cho thấy tỷ lệ nghiện sử dụng thiết bị này là $55,56 \%$. Tỷ lê này là cao so với tỷ lệ được báo cáo ở các nghiên cứu trên người trưởng thành ở Tây Ban Nha $(12,5 \%)$ và Bỉ $(21,5 \%)[4]$ và sinh viên Thụy Sĩ $(16,9 \%)$ khi sử dụng thang điểm SAS-SV [5]. Sự khác biệt này có thể một phần do sự khác biệt về sự phát triển công nghệ thông tin của Việt Nam nhứng năm gần đây [3].

Sinh viên là đối tượng có xu hướng nghiện điện thoại thông minh nhiều nhất [5]. Tỷ lệ sinh viển ở Hà Nội nghiện sử dụng điện thoại thông minh cao là một thực trạng đáng lo ngại và đây là dấu hiệu cảnh báo sự cần thiết phải xem xét các hậu quả có hại cho sức khỏe của nó ở cấp độ dân số [3].

4.2. Về giới tính và tuổi của sinh viên sử dụng điện thoại thông minh. Trong nghiên cứu hiện tại, sinh viên nữ tham gia chiếm tỷ lệ cao $(71,61 \%)$ và tỷ lệ sinh viên nữ nghiện điện thoại thông minh cũng chiếm tỷ lệ cao hơn sinh viên nam. Phát hiện này có phần trái ngược với quan điểm truyền thống là đàn ông dành thời gian vào công nghệ nhiều hơn phụ nữ. Tuy nhiên, thực tế đã chứng minh rằng phụ nữ thường sử dụng điện thoại thông minh với động cơ có liên quan đến vấn đề xã hội, trong khi nam giới thường sử dụng điện thoại thông minh với động cơ có liên quan đển những vấn đề thực tế và giải trí và việc đáp ứng các nhu cầu xã hội có thể mất nhiều thời gian hơn so với đáp ứng những nhu câuu thực tế. Những nghiên cứu trước đây cho thây rằng phụ nữ có một sự gắn bó mãnh liệt với điện thoại thông minh hơn so với nam giới [7]. Bển cạnh đấy, trong nghiên cứu của chúng tôi số sinh viên nghiện điện thoại thông minh chiếm tỷ lệ cao ở tuổi $18-20$ và số lượng sinh viên nữ ở tuối 25 nghiện điện thoại thồng minh cũng cao hơn so với sinh viển nam. Tỷ lệ cao này chỉ ra đối tượng vị thành niên thường có xu hướng nghiện điện thoai nhiều hơn những người tuổi lớn hơn, có thể do những người trẻ khả năng tự kiểm soát thấp hơn và dễ phụ thuộc vào điện thoại thông minh [5]. Tỷ lệ nữ giới nghiện điện thoại thông minh cao hơn so với nam giới cũng tương đồng với những nghiên cứu ở người Hàn Quốc và Trung Quốc [6], [8].

4.3. Về thời điểm bắt đầu sử dụng điện thoại thông minh và các ứng dụng thường dùng trên điện thoại thông minh. Các sinh viên đại học ở Hà Nội nghiện sử dụng điện thoại thông minh bắt đầu sử dụng thiết bị này ở tuổi 12-18 có số lượng lớn hơn so với số sinh viên không nghiện. Điều này phù hợp với những nghiên cứu gần đây cho thấy có mối quan hệ giữa những đối tượng bắt đầu sử dụng điện thoại thông minh sớm với lứa tuổi trưởng thành nghiện điện thoại thông minh. Mối quan hệ này cho thấy vai trò của gia đình và xã hội trong việc giáo dục trẻ em, giới hạn độ tuổi và thời gian sử dụng điện thoại thông minh nhằm góp phần làm giảm tỷ lệ nghiện điện thoại thông minh ở tuổi trưởng thành. Kết quả trong nghiên cứu này cũng chỉ ra rằng, phần lớn số sinh viên sử dụng ứng dụng Facebook và Facebook Messenger trong các ứng dụng được cài đặt trên điện thoại, điêu này cũng phù hợp với xu thế sử dụng các ứng dụng này của người Việt Nam [2].

4.4. Về tham gia thể dục thể thao và làm thêm của sinh viên sữ dung điện thoại thông minh. Trong nghiên cứu hiện tại cho thấy số lượng sinh viên nghiện điện thoại thông minh có tỷ lệ tham gia thể dục thể thao với tần suất "không bao giờ" và "thỉnh thoảng" cao hơn so với sinh viên không bị nghiện điện thoại thông minh. Những nghiên cứu gần đây cho thấy việc sử dụng điện thoại thông minh quá nhiều đã gẩy ra những triệu chứng về thể chất làm cho người sử dụng khó chịu [6]. Những khó chịu về thể 
chất cùng với việc dành thời gian quá nhiều vào việc sử dụng điện thoại thông minh có thể là nguyên nhân chính làm cho sinh viên nghiện điên thoại thông minh có tần suất tâp thể dục thể thao thấp hơn so với những sinh viên còn lại.

Số lượng sinh viên nghiện điện thoại thông minh có tỷ lệ làm thêm với tần suất "không bao giờ" và "thỉnh thoảng" cũng cao hơn so với sinh viên không bị nghiện điện thoại thông minh. Kết quả này phù hợp với báo cáo gần đây cho thấy sinh viên nghiện điện thoại thông minh dành quá nhiều thời gian cho việc sử dụng điện thoại, ảnh hưởng tới việc mất tập trung, không đạt được kết quả cao trong công việc. Mặt khác, điện thoại thông minh với các tín hiệu âm thanh và hình ảnh của chúng cảnh báo chủ sở hữu về các tin nhắn đến từ mạng xã hội, làm gián đoạn luồng suy nghĩ, tác động tiêu cực liên quan đến năng suất trong quá trình lao động. Những tác động trên dẫn đến tần suất và năng suất lao động giảm, vì vậy làm gián đoạn tham gia công việc làm thêm của sinh viên nghiện điện thoại thông minh.

\section{KẾT LUÂ̂N}

Trong số 1314 sinh viên năm 2-4 tại 36 trường đại học trên địa bàn thành phố Hà Nội, số sinh viên nữ sử dụng điện thoại thông minh nhiều hơn nam $(71,61 \%)$. Tỷ lệ sinh viên nghiện sử dụng điện thoại thông minh là $55,56 \%$. Lứa tuổi sinh viên sử dụng điện thoại thông minh chiếm tỷ lệ cao là 18-21 tuổi. Các ứng dụng sinh viên dùng phổ biến nhất trên điện thoại thông minh là Facebook và Facebook Messenger. Số lượng sinh viên nghiện điện thoại thông minh có tần suất tham gia thể dục thể thao và công việc làm thêm đều thấp hơn so với những sinh viển khác. Kết quả nghiên cứu này bước đầu cho thấy một số khía cạnh xã hội học của nghiện điện thoại thông minh ở sinh viên trên địa bàn Hà Nội là một thực trạng đáng lo ngại, là dấu hiệu cảnh báo cần thiết để xem xét các biện pháp nghiên cứu và can thiệp ở cấp độ rộng hơn.

TÀI LIẸU THAM KHẢO

1. Elhai JD, Dvorak RD, Levine, Hall, BJ, et al. (2017) "Problematic smartphone use: A conceptual overview and systematic review of relations with anxiety and depression psychopathology". Journal of Affective Disorders. 1(207): 251-259.

2. Kwon M, Kim DJ, Yang S, et al. (2013) "The Smartphone Addiction Scale: Development and validation of a short version for adolescents". PLoS One. 8(12): e83558.

3. APPOTA Technology for Change. Vietnam Mobile APP Market Report First half of 2018.
Available

https://appota.com/uploads/report/Vietnam_mobil e_app_market_Report_2018_EN.pdf $>$.

4. Lopez-Fernandez 0. (2017) "Short version of the Smartphone Addiction Scale adapted to Spanish and French: Towards a cross-cultural research in problematic mobile phone use". Addictive Behaviors. 64: 275-280.

5. Haug S, Castro RP, Schaub MP, et al. (2015) "Smartphone use and smartphone addiction among young people in Switzerland". Journal of Behavioral Addictions. 4(4): 299-307.

6. Hwang KH, Yoo YS, Cho OH. (2012) "Smartphone overuse and upper extremity pain, axiety, depression, and interpersonal relationships among college students". Journal of the Korea Contents Association, 12(10): 365-37.

7. Geser H. (2006) "Are girls (even) more addicted? Some gender patterns of cell phone usage". Sociology in Switzerland: Sociology of the Mobile Phone.

8. Luk TT, Wang MP, Shen $C$, et al. (2018) "Short version of the Smartphone Addiction Scale in Chinese adults: Psychometric properties, sociodemographic, and health behavioral correlates". Journal of Behavioral Addictions. 7(4): 1157-1165.

\section{ĐÁNH GIÁ CẢM GIÁC ĐAU VÀ NHẠY CẢM SAU ĐIỀU TRI CHE TỦY TRỰC TIẾP VỚI VẬT LIỆU SINH HỌC CALCIUM SILICATE}

\section{TÓM TẮT}

Mục tiêu: Mục tiêu nghiên cứu là đánh giá cảm giác đảau và nhạy cảm sau khi che tủy trực tiếp với

${ }^{1}$ Đai Hoc Y Dướ TP.HCM

Chịu trách nhiếm chính: Bùi Huỳnh Anh

Email: buihuynhanh@ump.edu.vn

Ngày nhân bài: 2/3/2021

Ngày phản biên khoa hoc: 1/4/2021

Ngày duyệt bài: 29/4/2021
Bùi Huỳnh Anh', Phạm Văn Khoa'

Mineral Trioxide Aggregate và Biodentine. Phương pháp: Thử nghiệm lâmm sàng với hai nhóm nghiên cứu đặt thuốc che tủy trực tiếp với Mineral Trioxide Aggregate và Biodentinetrên 22 răng cối nhỏ nguyên vẹn có chỉ định nhổ vì lý do chỉnh hình răng mặt ở các bệnh nhân từ 18-35 tuổi. Đánh giá cảm giác nhạy cảm hay đau sau điều trị sau 1, 7, 30 ngày với bảng câu hỏi và khám lâm sàng. Kêtt quả: Về cường độ và tỷ lệ đau, $64 \%$ nhóm MTA và $46 \%$ nhóm $B D$ bệnh nhân mô tả hiện tượng đau nhẹ và vừa. Về tổng thời gian đau, nhóm MTA có $29 \%$ trường hợp đau trong 1 ngày 